\title{
A systematic analysis of small supernumerary marker chromosomes using array CGH exposes unexpected complexity
}

\author{
Kavita S. Reddy, PhD1, Swaroop Aradhya, PhD², Jeanne Meck, PhD², George Tiller, MD', \\ Sridevi Abboy, MD ${ }^{1}$ and Harold Bass, MD ${ }^{1}$
}

Purpose: A small supernumerary marker chromosome is often seen in patients with developmental disorders. Prior to array-based comparative genomic hybridization markers were rarely genotyped end to end. In this study, a valid genotype-to-phenotype correlation was possible because the supernumerary marker chromosomes were fully characterized by array-based comparative genomic hybridization in a genome-wide analysis.

Methods: Ten consecutive de novo small supernumerary marker chromosome cases were systematically genotyped using G-banding, C-banding, AgNOR staining, whole-genome array-based comparative genomic hybridization, and fluorescence in situ hybridization.

Results: Among 10 small supernumerary marker chromosome cases studied, 4 (40\%) were not identified by array-based comparative genomic hybridization because of low-level mosaicism or because they lacked euchromatin. One case $(10 \%)$ was a simple pericentromeric marker extending from 5 p13.3 to $5 q 11.2$. Five (50\%) markers showed unexpected complexity. Two cases had markers that were derivative acrocentric (AgNOR+) chromosomes with the euchromatin from chromosomes 18p or 19p. Each of the other three cases with complex markers had unusual characteristics including a marker from noncontiguous segments of chromosome 19q, a highly complex rearrangement involving a chromosome 20 homolog as well as the small supernumerary marker chromosome, and a mosaic duplication of a proximal 8p marker.

Conclusion: Small supernumerary marker chromosomes are frequently complex on the basis of our small sample. Whole-genome array-based comparative genomic hybridization characterization of the small supernumerary marker chromosome provided informed genetic counseling.

Genet Med 2013:15(1):3-13

Key Words: array CGH; complex marker chromosomes
Small supernumerary marker chromosomes (sSMCs) are chromosomal fragments or markers whose origins often cannot be determined by conventional cytogenetic methods alone and require molecular approaches for definitive characterization. sSMCs are, in general, equal in size or smaller than a chromosome 20 of the same metaphase spread. Complex SMCs are composed of noncontiguous DNA or amplicons. SMCs are found in $\sim 0.043 \%$ of live births and $\sim 0.075 \%$ prenatal cases and are seven times more prevalent in intellectually disabled patients. ${ }^{1}$ Approximately $77 \%$ of SMCs are de novo and $23 \%$ are inherited, either from the mother (16\%) or the father (7\%). A ring-like SMC occurs with a frequency of $0.14-0.72 / 1,000$ in newborns and $0.14-1.5 / 1,000$ in prenatal diagnoses. ${ }^{2}$ Most SMCs (70\%) are derived from the short arms and pericentromeric regions of acrocentric chromosomes. Those derived from nonacrocentric autosomes are rare and occur with a frequency of $\sim 15 \%$ of all markers. ${ }^{3}$ Markers that are not derived from acrocentric chromosomes are often suspected to be small ring chromosomes on the basis of their morphology and behavior. ${ }^{4}$ Whole-genome array-based comparative genomic hybridization (aCGH) and fluorescence in situ hybridization (FISH) can now provide accurate characterization of SMCs in terms of chromosomal origin, gene content, uniparental disomy, and other concomitant imbalances elsewhere in the genome. Without the benefit of aCGH, the empiric risk of phenotypic abnormalities associated with a prenatally detected de novo SMC is $18 \%$ if it contains satellites and $31 \%$ if it does not have satellite sequences. ${ }^{2}$ This risk depends on a number of factors, including ultrasound findings, whether the SMC is familial, and if it is associated with a known syndrome. Certain marker chromosomes are consistently identifiable by G-banding and have a well-established phenotype. Examples include isochromosome 12p, associated with Pallister-Killian syndrome (OMIM 601803) and isochromosome $18 \mathrm{p}$, which causes mild-moderate mental retardation and a characteristic facial appearance. ${ }^{5}$ For chromosome 15 -derived marker chromosomes, often seen as isodicentric 15q, FISH analysis allows discrimination between large markers that contain the SNRPN locus (OMIM 182279) and are tetrasomic for the Prader-Willi syndrome (OMIM 176270) or Angelman syndrome (OMIM 105830) critical region and those small markers that do not contain SNRPN. Based on the parental origin, the larger markers are known to cause a phenotype involving 
some combination of mental retardation, seizures, autistic features, and growth retardation, whereas the latter are usually associated with a normal phenotype. ${ }^{6-8}$ Similarly, FISH analysis of chromosome 22-derived markers can reveal whether the SMCs contain the critical region for cat eye syndrome (OMIM 115470 ), which is characterized by ocular coloboma and other dysmorphic features. ${ }^{9}$ However, our knowledge on the clinical significance of the other sSMCs is limited. Although complex markers are estimated to form only a small percentage $(\sim 0.9 \%)$ of SMCs, ${ }^{10}$ this may be an underestimation as highlighted in two recent studies applying aCGH to SMCs. ${ }^{11,12}$ To address the frequency of complex markers and to provide prognosis based on a meaningful genotype-to-phenotype correlation, we took a systematic, comprehensive approach to precisely characterize 10 consecutive SMCs using chromosome analysis, aCGH, and FISH.

\section{MATERIALS AND METHODS}

Whole-genome aCGH was performed using a custom-designed oligonucleotide array with $\sim 180,000$ probes. The nonrepetitive sequence of the human genome was covered with probes spaced $35 \mathrm{~kb}$ apart, on average. More than 200 clinically significant regions had a higher probe density with $250 \mathrm{bp}-5 \mathrm{~kb}$ probe spacing. The array was designed based on human genome build hg19/GRCh37. For the aCGH, $0.5 \mu \mathrm{g}$ of patient DNA was labeled with Cy5 dye and a sex-matched reference DNA (Promega, Madison, WI) was labeled with Cy3 dye. After purification, the labeled products were combined and hybridized to the custom array for $40 \mathrm{~h}$ at $65^{\circ} \mathrm{C}$. The arrays were washed and scanned on a $3-\mu \mathrm{m}$ scanner to obtain a TIFF image. This image was imported into Feature Extraction software to quantify the fluorescence data. The resulting data were imported into Agilent Genomic Workbench 6.5.0.25 software for visualization of copy number genome-wide. FISH using custom-labeled BAC probes
(Empire Genomics, Buffalo, NY) and commercial centromeric probes (Abbott Molecular, Des Plaines, IL) was performed following the standard protocol.

For those cases in which genotyping was performed to assess parental origin of the sSMC, we used an array that contained the same probes for copy-number analysis and also 66,480 oligonucleotide probes that contained single-nucleotide polymorphisms. These single-nucleotide polymorphisms were assayed for zygosity by cutting with AluI and RsaI restriction enzymes. The DNA $(0.5 \mu \mathrm{g})$ from the patient and from a sex-matched HapMap sample (European) was digested with AluI and RsaI for $3 \mathrm{~h}$. The digested fragments were purified, labeled, and arrays processed as described earlier. The IRB approval can be found in Supplementary material online.

\section{RESULTS AND DISCUSSION}

Table 1 summarizes the results from the systematic use of conventional cytogenetic banding methods ( $G, C$, and AgNOR), aCGH analysis, and FISH studies. The morphology of sSMC from the banding methods and precise genomic content defined by aCGH analysis determined the FISH probes necessary for the complete characterization of the marker. Based on our findings, the markers are classified into three groups: marker unidentified by aCGH, simple marker, and complex marker.

\section{Markers unidentified by aCGH}

Case 1. Figure 1a represents a small mosaic bisatellited marker present in $93 \%$ of cells by G-banding, which was not detected by aCGH. Therefore, this marker was assumed to lack euchromatin. About two-thirds of reported sSMCs were derived from acrocentric chromosomes; almost $70 \%$ of those acrocentric-derived sSMC were inverted duplications or dicentric derivatives without euchromatin and were transmitted through several generations in some families. ${ }^{13}$

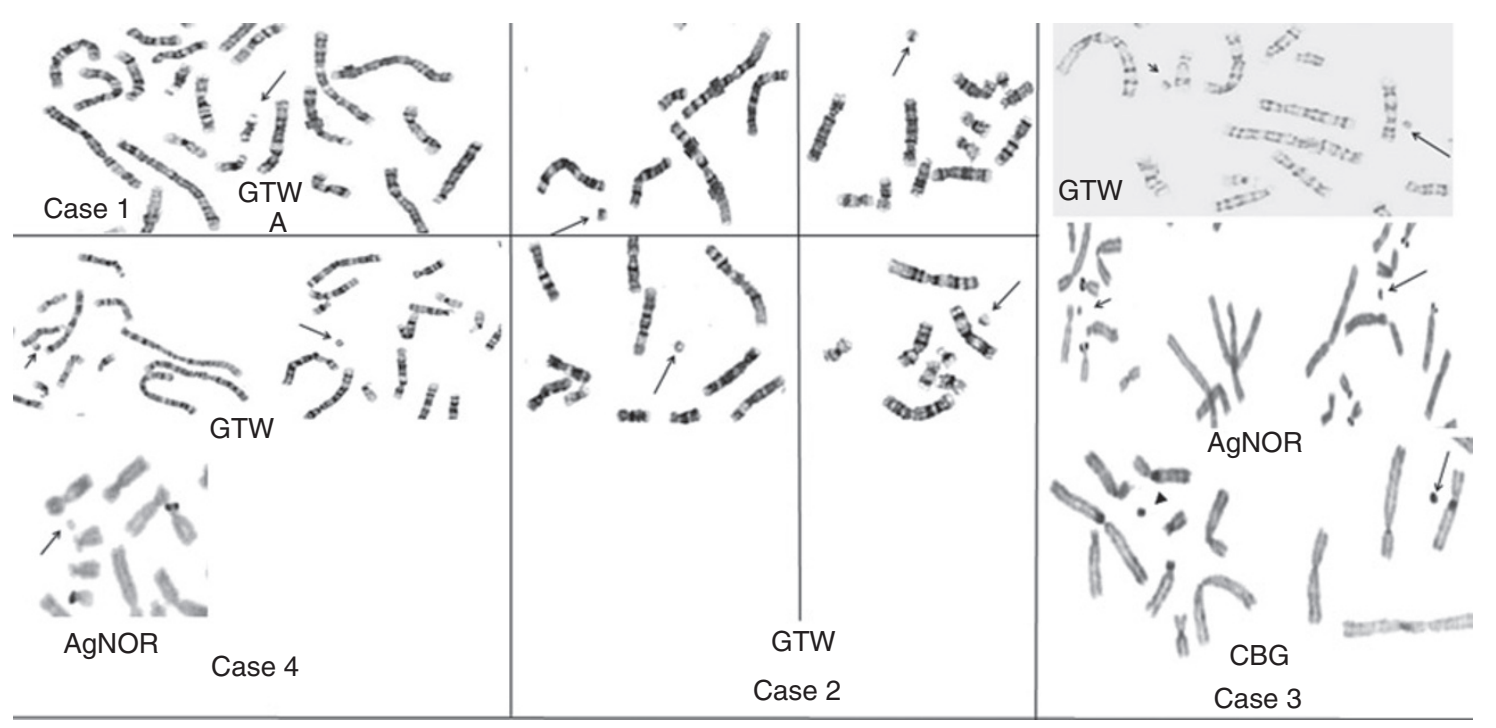

Figure 1 Markers not identified by aCGH. (a) Case 1: A bisatellited G-banded marker (arrow). (b) Case 2: Four partial metaphases with markers (arrows) that appear ring-like. (c) Case 3: Two markers: Marker 1, C-band- and AgNOR-positive (arrow); marker 2, only C-band-positive (arrowhead) and ring-like. (d) Case 4: Marker is ring-like and AgNOR-negative (arrow). GTW, G-bands after trypsin pretreatment and Wright stain. 

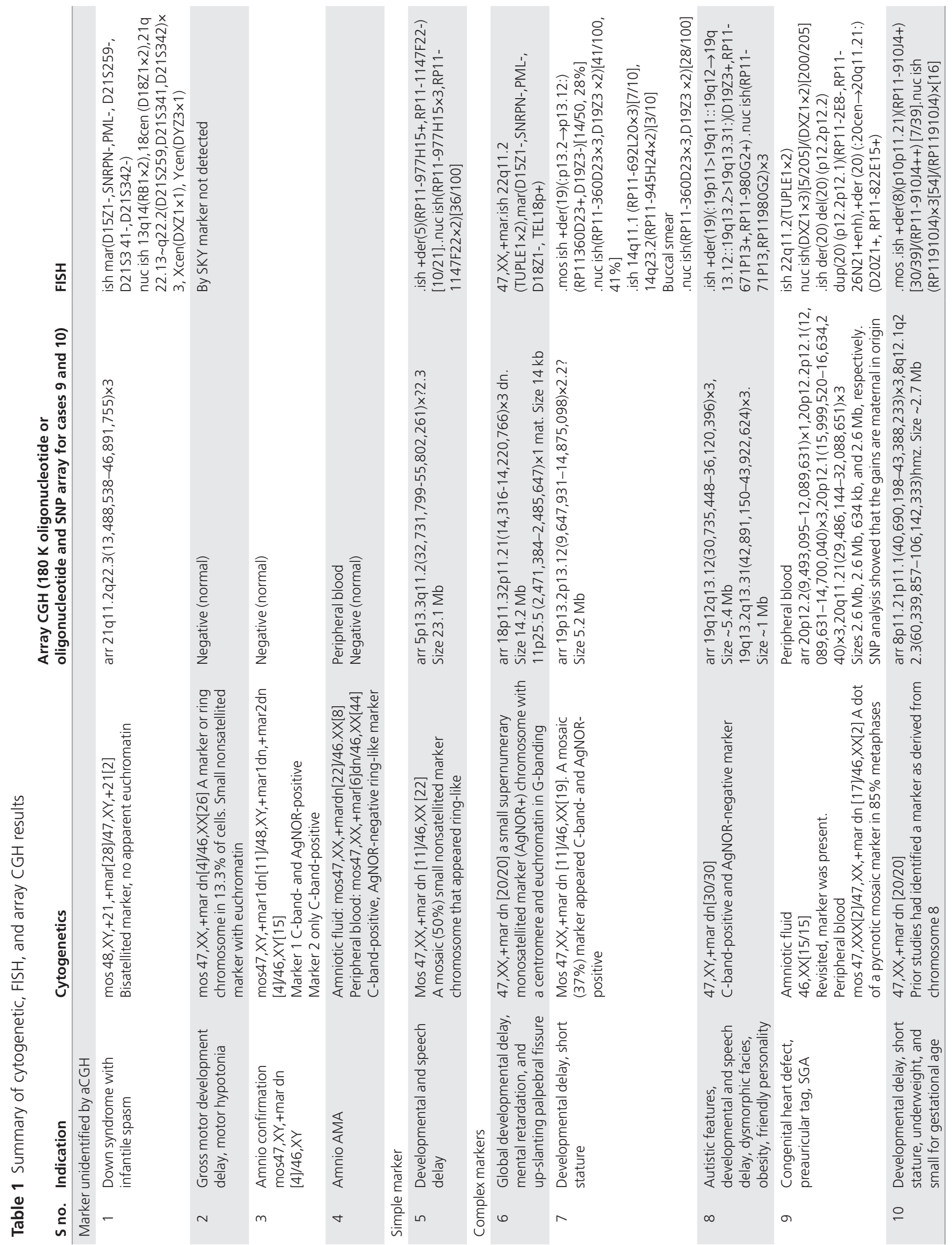
Case 2. (Figure 1b) A mosaic (13.3\%), small, nonsatellited, ring-like marker chromosome with euchromatin was not detected by aCGH or spectral karyotyping.

Case 3. (Figure 1c) Two cell lines were present: one with a marker 1 in 37\% cells and the other with markers 1 and 2 in $13.3 \%$ cells, but neither were detected by aCGH. Marker 1 was C-band- and AgNOR-positive, and marker 2 was only C-bandpositive and ring-like.

Case 4. (Figure 1d) A mosaic (12\%), nonsatellited, ring-like marker with a centromere was not detected by aCGH.

Markers in cases 2-4 may not have been detected by aCGH because of the low percentage of cells with the marker.

\section{Simple marker}

Case 5. (Figure 2) A mosaic (50\%), de novo, small, nonsatellited marker chromosome that appeared ring-like in G- and
C-banding was found in a patient with developmental and speech delay. aCGH showed a gain of $23.1 \mathrm{Mb}$ in $~ 30 \%$ of cells for the region 5p13.3q11.2 (chr5:32,731,799-55,802,261). By FISH analysis, probe RP11-977H15 [5p13.1] localized to the supernumerary marker in $48 \%$ of metaphases and gave an additional signal in $36 \%$ of interphases. The marker included the reported 5 p13 duplication syndrome (OMIM 613174) associated with developmental delay, mental retardation in all patients, and other features in some patients, while 5q11 trisomy has been associated with learning difficulties and speech delay. ${ }^{14}$

\section{Complex markers}

Case 6. (Figure 2) aCGH showed a de novo $14.2 \mathrm{Mb}$ gain of the short arm of chromosome 18p11.32p11.21 in a patient with global developmental delay, mental retardation, and upslanting palpebral fissures. A G-band SMC was monosatellited,

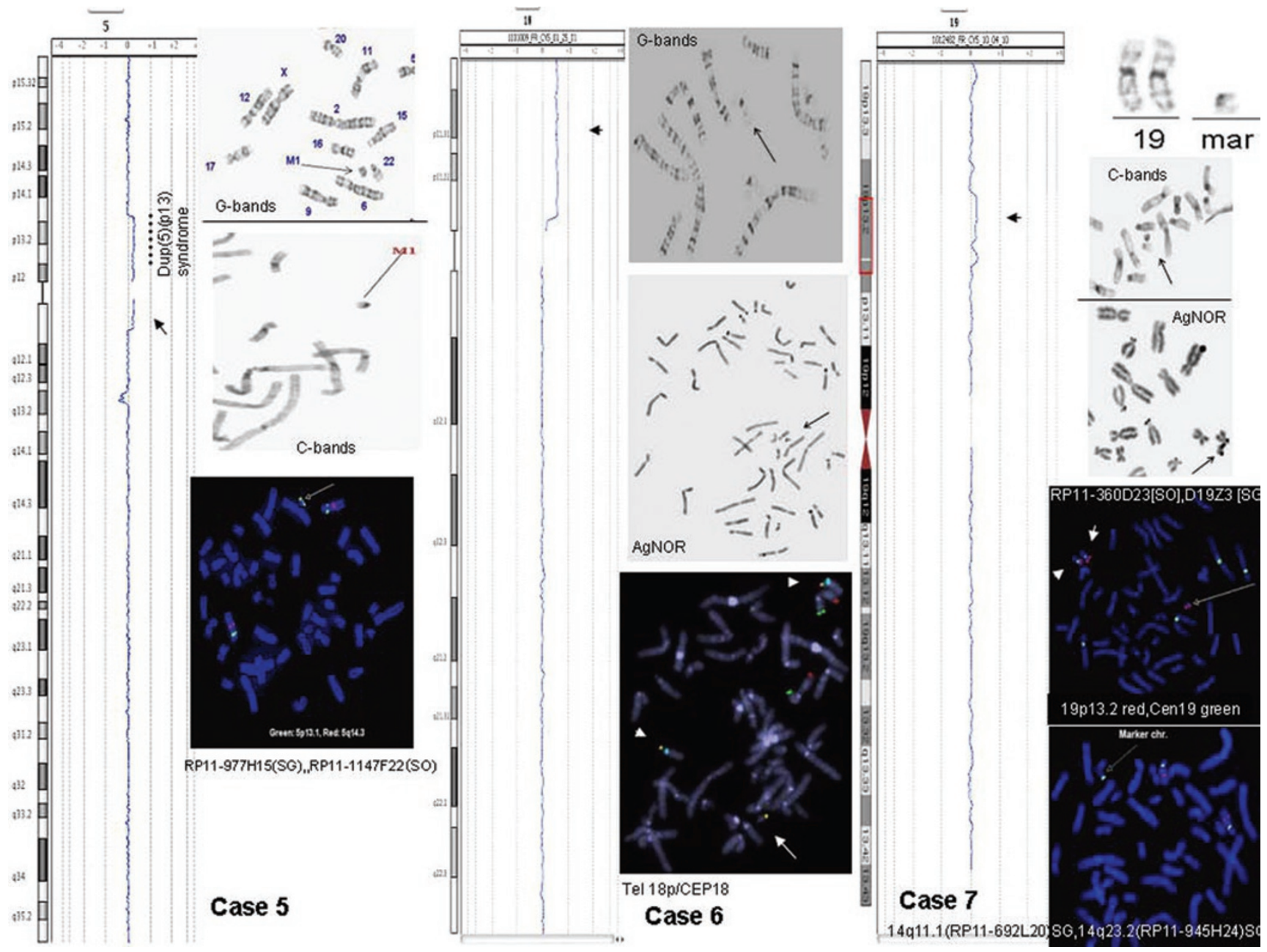

Figure 2 Case 5: aCGH shows a gain of 5p13.3q11.2(32,731,799-55,802,261)×?2.3 of size $23.1 \mathrm{Mb}$ in $\sim 30 \%$ of cells (arrow). Of note is that gain of this region is pathogenic and associated with a 5 p13 duplication syndrome in the OMIM phenotype track. A small and nonsatellited marker chromosome appears ring-like in G- and C-banding (arrows). FISH confirmation showed a 5p13.1 signal on the marker (arrow). Case 6: aCGH shows gain of the short arm of chromosome 18:14,316-14,220,766 of size $14.2 \mathrm{Mb}$. A G-band monosatellited marker (arrow) is positive for AgNOR-staining (arrow). FlSH shows the marker is 18ptel-positive (arrow) and negative for 18 centromere (arrow) using telomere $11 \mathrm{p} / 11 \mathrm{q} / 18 \mathrm{p}$ and CEP18 probes. Case 7: aCGH shows a gain of 19p13.2p13.12(9.647,931-14,875,098)×2.2?. A G-banded, CBG + and AgNOR+ stained marker (arrow). FISH using RP11-360D23 [SO](19p13.2) and D19Z3 [SG](19 centromere) hybridized to the two normal 19 chromosomes (arrowhead) and only RP11-360D23 hybridized to the marker (arrow). Probe RP11-692L20 (14q11.1) hybridized to the marker (arrow) and control RP11-945H24 (14q23.2) probe was on the normal 14s but not on the marker. 
positive for silver staining, and negative for 18-centromere FISH probe. Hence, the SMC was derived from a translocation between an acrocentric chromosome (other than chromosome 15) and chromosome 18 short arm (18ptel-positive by FISH). Trisomy of the 18p arm causes variable intellectual disability, developmental delay, minor facial dysmorphism, and other anomalies. ${ }^{15}$ In addition, our proband had a maternally inherited $14 \mathrm{~kb}$ deletion in the KCNQ1 gene on 11p25.5. Mutations in KCNQ1 cause long QT syndrome (OMIM 192500). ${ }^{16,17}$ Although the deletion included the first exon of the KCNQ1 gene in the minor transcript NM_181798, the expression pattern and prevalence of this transcript are unknown. In the commonly used reference transcript for KCNQ1, NM_000218, the small deletion falls within an intron. ${ }^{18}$ These results were also confirmed by targeted exon-level aCGH. The maternal sample showed the 11p25.5 deletion and normal copy number for 18p11. The paternal sample showed normal results for both genomic regions. The clinical significance of the deletion within KCNQ1 is currently unknown.

Case 7. (Figure 2) A de novo, satellited, mosaic (37\%) sSMC had a C-band and was AgNOR-positive. aCGH showed a mosaic (20\%) 5.2 Mb gain of chromosome 19p13.2p13.12. This gain was shown to be present on the SSMC using probes RP11360D23 and RP11-360D23 that map to chromosome 19p13.2. The marker was shown to be derived from chromosome 14 by FISH using the RP11-692L20 [14q11.1] probe and was shown to be negative for the D19Z3 centromere probe. FISH analysis detected mosaicism for the marker in $28 \%$ of metaphases and in $41 \%$ of interphases. This $5.2 \mathrm{Mb}$ genomic region contains a large number of genes, including several that are associated with clinical disorders. OMIM-listed genes in this interval are TYK2, DNM2, LDLR, EPOR, PRKCSH, MAN2B1, RNASEH2A, $G C D H, L Y L 1, C A C N A 1$, and CC2D1A.

The proband, with developmental delay, was in fourth-grade special education and had speech therapy for difficulty with language, such as putting words together. She was unable to state her birthday. A review of systems revealed absence of seizures. Physical examination showed the following results: blood pressure 119/60; pulse 83; height $1.165 \mathrm{~m}$ (3 feet 9.87 inches); weight $26.4 \mathrm{~kg}$ (58 lb $3.2 \mathrm{oz}$ ), and head circumference $49 \mathrm{~cm}$. Growth percentiles based on body mass index for age, stature for age, and OFC for age were $85.13 \%\left(19.45 \mathrm{~kg} /\left(\mathrm{m}^{2}\right)\right), 0.11 \%$ ( $50 \%$ for 6.5 years) and $20 \%$, respectively. Facial features were remarkable for periorbital fullness (similar to parents), somewhat arched eyebrows, mild synophrys, somewhat smooth philtrum, and prominent ears with attached lobes. Skin was without rashes, lesions, or stria distensae. Of note were short stature and developmental delay. However, both parents and siblings were short. The parents were of advanced age when the proband was conceived.

The reported phenotype associated with gains that include the chromosome 19p13.2p13.12 region (chr19:9,647,931$14,875,098$ ) is summarized in Supplementary Table S1, online. A nonmosaic duplication involving the 19p13.2p13.13 genomic region was described in an individual with developmental delay, microcephaly, heart defects, and multiple minor anomalies. ${ }^{19}$ Another 12-month-old infant was evaluated for severe hypotonia, psychomotor retardation, and facial dysmorphisms that included round face, high prominent forehead, downwardslanted palpebral fissures, hypertelorism, short nose, chubby cheeks, long philtrum, anteverted lower lip, and low-set asymmetric and dysmorphic ears. Karyotype analysis disclosed an extra mosaic ring chromosome, which included the whole 19p $\mathrm{arm} .^{20}$ The clinical finding in one patient with an SMC for chromosome 19p was developmental delay. ${ }^{21}$ A 9-year-old boy with psychomotor retardation had a small mosaic sSMC. The karyotype was interpreted as: $47, \mathrm{XY},+$ mar.ish $\operatorname{der}(19)(: \mathrm{p} 13.3 \rightarrow \mathrm{p} 11:)$ $(839 \mathrm{~B} 1+, 872 \mathrm{G} 3+, 728 \mathrm{C} 8+, \mathrm{D} 1 / 5 / 19 \mathrm{Z}+) \operatorname{dn}[52] / 46, \mathrm{XY}[48] .{ }^{22} \mathrm{~A}$ microduplication for 19p13.2 (chr19:9,109,407-11,068,542) by aCGH presented with variable neurocognitive disability, overgrowth, and facial dysmorphism similar to Sotos syndrome. Increased expression of duplicated genes ILF3 (interleukin enhancer-binding factor 3), ZNF266 (zinc finger protein 266), DNMT1 (DNA methyltransferase gene 1), and SMARCA4 (Swi/Snf-related, matrix-associated, actin-dependent regulator of chromatin, subfamilyA, member 4) in peripheral blood, seen by whole-genome expression array transcript level and quantitative PCR, supported gene dosage as the cause for this disorder. ${ }^{23}$

Case 8. (Figure 3) A de novo sSMC was C-band-positive and AgNOR-negative. aCGH displayed gains in 19q12q13.12 (chr19:30,735,448-36,120,396) of $\sim 5.4 \mathrm{Mb}$ in size and in 19q13.2q13.31 (chr19: 42,891,150-43,922,624) of $\sim 1 \mathrm{Mb}$ in size. The chromosome 19 centromere and both gains were shown to be on the marker by FISH using D19Z3, RP11-671P13 [19q13.1], and RP11-9 80G2 [19q13.2] probes.

A child born to parents of advanced age was seen for developmental delay and obesity. The patient started walking at 9 months, spoke the first word at 6 months, began using sentences at 18 months, and was toilet trained. There was a history of regression. At 3 years of age, he was diagnosed with autism and has not been re-evaluated. He reportedly has an individual education program/plan and was in the second grade, receiving speech therapy every day for $30 \mathrm{~min}$ and adaptive physical education. On examination, the proband was friendly and made good eye contact. Speech impairment was observed when his brother was born (around 18 months of age) and then again at around 4 years of age. He has been in special education since kindergarten and can recognize a few letters of the alphabet and reportedly can do basic math. There is no family history of problems similar to the patient's condition.

Physical examination showed $99.28 \%$ of growth percentile based on weight for age ( $+3 \mathrm{SD}$ above the mean) and occipitofrontal circumference of $53 \mathrm{~cm}$ (75\%). Habitus was obese. $\mathrm{He}$ had upswept anterior hairline, low posterior hairline, and a widow's peak. Mid-occipital flatness, up-slanted palpebral fissures, and medially sparse eyebrows were also present. Other features were widely spaced teeth, smooth philtrum, thin upper lip, a short neck, and gynecomastia. Bilaterally, thumbs were short; he also had short fifth fingers with clinodactyly, brachydactyly, 

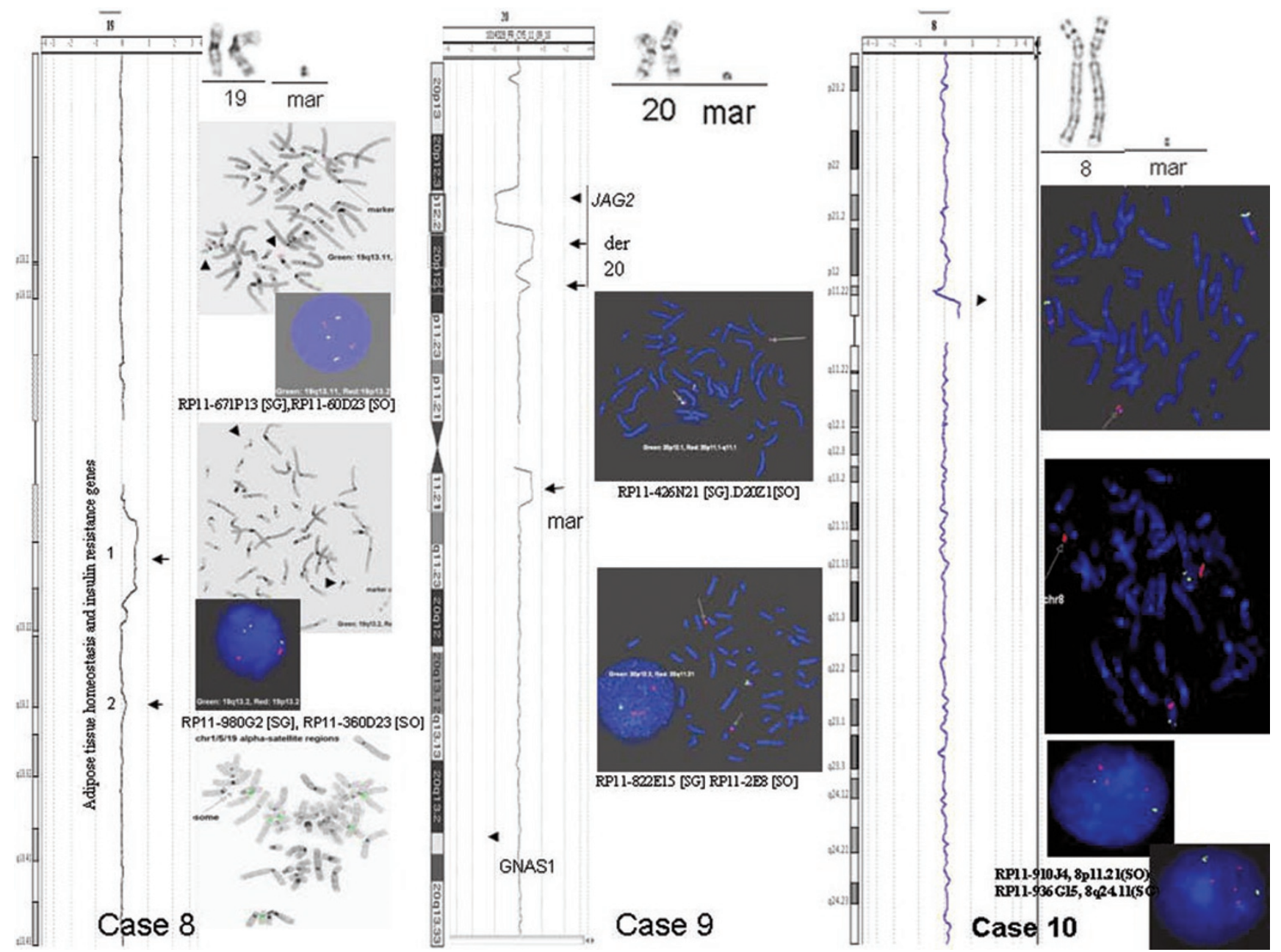

Figure 3 Case 8: array-based comparative genomic hybridization (aCGH) shows a gain of $~ 5.4 \mathrm{Mb}$ from chromosome 19q12q13.12 (arrow). In addition, there is a gain of at least $1 \mathrm{Mb}$ from chromosome band 19q13.2q13.31 (arrow), including at least three adipose tissue homeostasis and insulin resistance genes (http://genome.ucsc.edu, HG 19). A partial G-banded karyotype of chromosome 19 pair and the marker. FISH using probes RP11-671P13 from chromosome 19q13.11 and probe RP11-980G2 from chromosome 19q13.2 hybridized to the marker (gray arrows) and gave three signals in interphases. The centromere of chromosome 19 probe showed an additional FISH signal on the supernumerary marker chromosome (gray arrow). Case 9: aCGH shows a complex set of rearrangements on chromosome 20 (arrows). The first imbalance is a $2.6 \mathrm{Mb}$ deletion of virtually the entire 20p12.2 band involving JAG2 associated with Alagille syndrome (arrow). The second imbalance is a $2.6 \mathrm{Mb}$ duplication of 20p12.2p12.1 (arrow). There is also a $634 \mathrm{~kb}$ duplication in 20p12.1 (arrow). Finally, there is a $2.6 \mathrm{Mb}$ duplication in the pericentromeric region of the long arm of chromosome $20 \mathrm{q} 11.21$ (arrow). A partial karyotype of chromosome 20 pair and the marker chromosome. Metaphase FISH performed with probes targeted to 20p12.1 (RP11-426N21) [SG], 20p12.2 (RP11-2E8), [SG], 20q11.21 (RP11822E15) [SO], and the centromere (D20Z1) [SO] showed that this patient carries a derivative chromosome 20 with a deletion in 20p12.2 (lower metaphase, short arrow). Furthermore, the 20p12.1 duplication by array was seen as a larger signal (upper metaphase, short arrow) in metaphases and was confirmed in interphases to be a tandem duplication. The centromere on the supernumerary marker chromosome was derived from chromosome 20 (upper metaphase, long arrow). The imprinted GNAS gene is not included in the marker. Case 10 A SMC was from 8p11.1p11.2 seen by aCGH (arrow) and confirmed by RP11-910J4 [SO] (8p 11.2) as a smaller or a larger duplicated signal in metaphases and as 3 or 4 red signls in interphases. SG, spectrum green; SO, spectrum orange.

and tapered fingers. Fetal fat pads were present. He had developmental delay, speech difficulties, and hyperactivity.

Quack et al. ${ }^{24}$ reported a supernumerary ring chromosome derived from the proximal part of the long arm of chromosome 19 [47,XY,+ $\mathrm{r}(19)(\mathrm{q} 11-\mathrm{q} 13.2)]$. The patient presented with mental retardation and macrocephaly, and weight was reported to be three standard deviations above the mean. He was born small for gestational age, and had some dysmorphic features (hypertelorism, anti-Mongolian slants, and minor nose and mouth malformations). His mother and sibling were normal and carried the same ring chromosome but had a balanced karyotype with an interstitial deletion of the long arm of chromosome 19. Zung et al. ${ }^{25}$ described a 13-year-old with a marker consisting of 19q12q13.2, constitutional obesity, mental retardation, and dysmorphic features. Because this region contains several genes that are related to adipose tissue homeostasis and insulin resistance, several metabolic comorbidities such as insulin resistance, dislipidemia, and hyperuricemia may be associated with this syndrome. The morbid obesity observed began as overweight, but from 5 years there was rapid gain in body weight, although the patient was not hyperphagic. Davidsson et al. ${ }^{26}$ presented a girl with 
two supernumerary marker chromosomes causing a duplication of $19 q 12 q 13.2$, with delayed developmental milestones, corpus callosum anomalies, microphthalmia, and obesity. The $19 \mathrm{q}$ segment contained the genes AKT2, CEACAM1, CEBPA, $L I P E$, and TGFB1, which are involved in adipose tissue homeostasis and insulin resistance and that could potentially have contributed to the obese phenotype observed. Hall et al. ${ }^{27}$ also described a patient who had obesity and macrocephaly and a supernumerary de novo mosaic marker involving cytoband 19q13.11q13.2. Our obese proband also has gains of CEACAM1, CEBPA, LIPE genes due to the SMC. This obesityassociated genetic syndrome could have implications in the management and treatment of patients with a duplication of 19q12q13.2.

Case 9. (Figure 3) High-resolution chromosome analysis on peripheral blood revealed a 46,XX karyotype in 10\%, 47,XXX in $10 \%$, and a nonsatellited sSMC in $80 \%$ of cells. The karyotype was mos47,XXX[2]/47,XX, +mar[17]/46,XX[2]. Parental chromosome analysis showed a 46,XY[50] complement in the father and low-grade 45,X (6\%) mosaicism in the mother, who was of advanced maternal age. Neither parent was found to carry a marker chromosome. Prenatal chromosome analysis on amniotic fluid was reported as normal; these slides were re-evaluated and the marker was identified. aCGH showed a complex set of rearrangements involving chromosome 20. The first imbalance was a $2.6 \mathrm{Mb}$ deletion of virtually the entire 20p12.2 band; the second imbalance was a $2.6 \mathrm{Mb}$ duplication of 20p12.2p12.1; the third was a $634 \mathrm{~kb}$ duplication in 20p12.1; and the final imbalance was a $2.6 \mathrm{Mb}$ duplication of the pericentromeric region of the long arm of chromosome 20q11.21. Mosaicism was not evident for any of the copy-number changes. When polymorphic markers were informative, genotype analysis showed only paternal alleles in the deleted segment and extramaternal alleles within the duplicated segments. Metaphase FISH performed on 25 cells with probes targeted to 20p12.1 (RP11-426N21), 20p12.2 (RP112E8), 20q11.21 (RP11-822E15), and the centromere (D20Z1) showed that this patient carries a derivative chromosome 20 with a deletion in 20p12.2 (RP11-2E8) and tandem duplication 20p12.2-20p12.1 (RP11-426N21). The latter was confirmed in interphase FISH. In addition, the supernumerary marker chromosome showed a chromosome 20 centromere (D20Z1) and the pericentromeric region of 20q11.21 (RP11-822E15), which corresponded to the $2.6 \mathrm{Mb}$ gain detected by aCGH.

The deletion in 20p12.2 affects seven genes, including JAG1. Haploinsufficiency for JAG1 causes Alagille syndrome, which involves posterior embryotoxon in the eyes, bile duct paucity, congenital heart defects, butterfly vertebrae, typical facies, and other anomalies. ${ }^{16,17,28}$ In addition to JAG1, MKKS is deleted in our patient. Mutations in $M K K S$ lead to autosomal recessive McKusick-Kaufman syndrome (OMIM 236700) or Bardet-Biedl syndrome type 6 (OMIM 209900). The $2.6 \mathrm{Mb}$ duplication in 20p12.2p12.1 affects eight genes. Among these, C20orf7 is associated with autosomal recessive mitochondrial complex I deficiency (OMIM 252010), and the others are not known to be clinically relevant at present. Sections of this 2.6
$\mathrm{Mb}$ genomic region are reported to vary in copy number in the normal population.

The $634 \mathrm{~kb}$ duplication in 20p12.1 is a likely benign copynumber change because it affects only the MACROD2 and KIF16B genes; these genes are not known to be clinically significant and parts of their sequences are reported to vary in copy number in the normal population. ${ }^{29}$ The last genomic imbalance in this patient is a $2.6 \mathrm{Mb}$ duplication of $20 \mathrm{q} 11.21$ in the pericentromeric region of the long arm that corresponds to the marker identified in this patient's karyotype. This duplicated region contains more than 35 genes, including a cluster of the $D E F B$ (defensin) genes. This particular duplication has not been previously described, but a larger duplication, $20 \mathrm{q} 11.21 \mathrm{q} 12$, has been reported to result in psychomotor retardation, craniofacial anomalies, and a severe vision deficit. ${ }^{30} \mathrm{~A}$ second report describes a supernumerary chromosome 20 with material from 20 p11.21 to $20 \mathrm{q} 11.21$ in a patient with a normal phenotype and obesity. ${ }^{31}$

Our proband was born to a nonconsanguineous couple of advanced parental age. The couple's only other pregnancy together was electively terminated for fetal hydrocephalus; amniocentesis revealed a normal karyotype. In addition, through a previous relationship, the mother had two healthy offspring. During the proband's pregnancy, the mother was treated with insulin for gestational diabetes. There was poor fetal growth in the third trimester, but maternal titers for cytomegalovirus, herpes simplex, rubella, syphilis, and toxoplasmosis were negative. A cesarean section was performed at 35 weeks' gestation for intrauterine growth restriction and oligohydramnios. The infant weighed $1,995 \mathrm{~g}$ (10th centile), was $39.5 \mathrm{~cm}$ long (less than third centile), and had a $30.5-\mathrm{cm}$ head circumference (10th centile). There were three pedunculated, preauricular skin tags on the left. Cranial and renal ultrasound studies were normal. The infant passed newborn screening and was discharged on day 8. A heart murmur due to pulmonary valvular stenosis was detected at 2 weeks of age. The infant underwent a percutaneous pulmonary valvuloplasty at 6 weeks of age. When examined at 8 months of age, weight, length, and head circumference were symmetrically below the second centile. The forehead was broad, previously described preauricular tags were present, ears were borderline low-set with over-folded helices and a deep vertical groove behind the right ear helix, the nasal bridge was broad, the upper lip was thin with a slightly downturned mouth and normal palate, and a grade 3 of 6 harsh systolic murmur could be heard over the precordium. Tone was increased without scissoring. The infant reached for toys, used consonants, and imitated sounds, but was unable to roll over from the back to the abdomen and could only sit when brought into position. There was no jaundice or hepatomegaly, and the parents reported normal stools. Eye examination by a pediatric ophthalmologist revealed no posterior embryotoxon or other anomalies. Liver enzymes and bilirubin were normal. Spine films demonstrated butterfly-type vertebrae T10 and T11. X-rays of the chest and ribs were normal. 
Table 2 Reported complex markers examined using aCGH

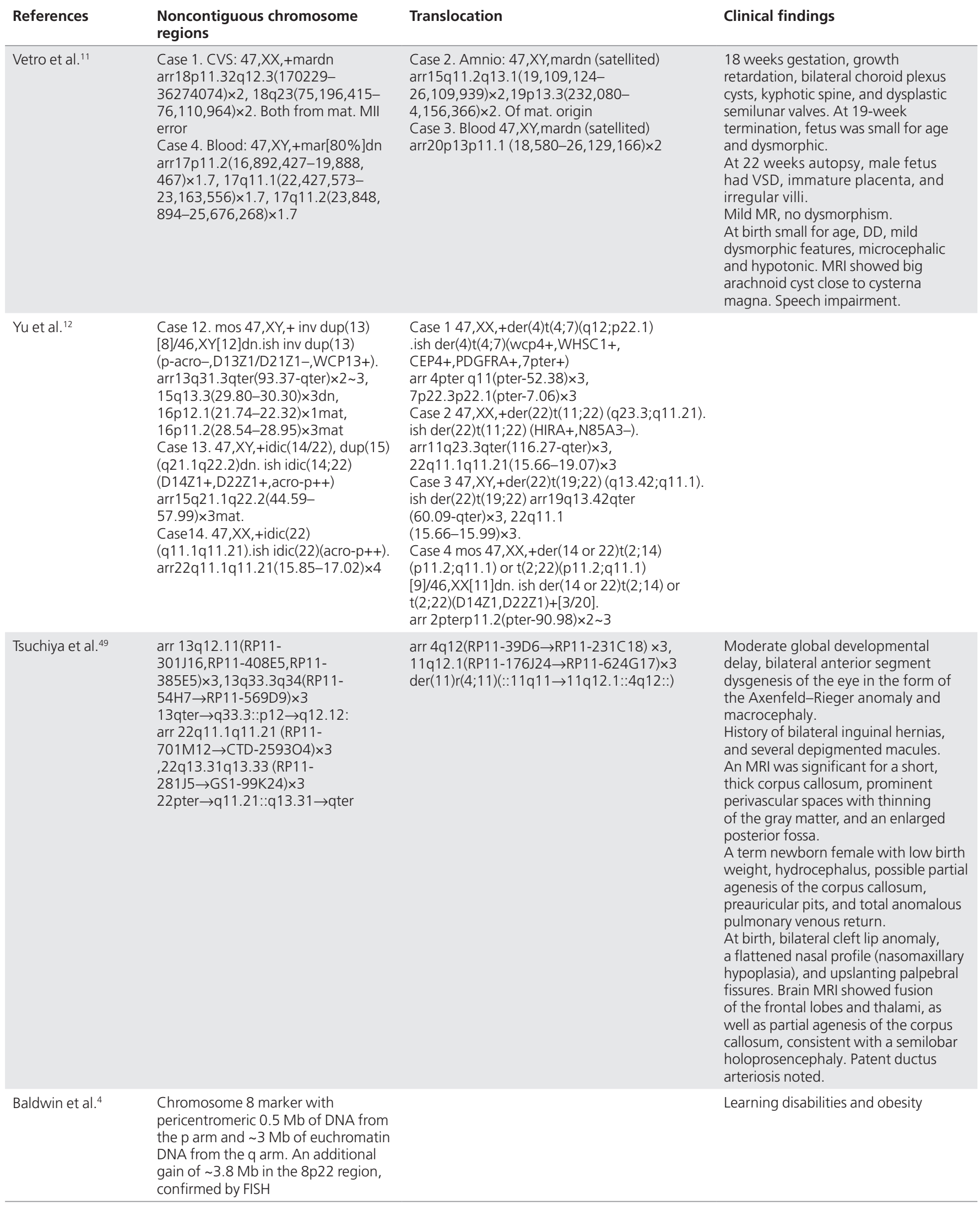


Supernumerary marker/ring chromosome 20 is a rare abnormality and no common phenotype has been described. Because the majority of previous reports of extra marker/ring 20 have not been sized, a genotype-phenotype relation is difficult to establish. Guediche et al. ${ }^{31}$ reviewed the supernumerary ring chromosome 20 and surmised varying degrees of phenotypic abnormality. Among these descriptions, the most frequently noted manifestations were growth and psychomotor retardation, low-set ears, hand and foot anomalies, and micrognathia. ${ }^{32-40}$ In patient $2,{ }^{30}$ a supernumerary marker was found in $43 \%$ of amniocytes and fetal lymphocytes. At 15 months of age, except for obesity, her psychomotor development was normal and no dysmorphic features were noted. In this patient, the proximal breakpoint on the short arm of chromosome 20 was located between nondeleted RP5-1025A1 and deleted RP1-234M6 (20p11.21) and on the long arm between nondeleted RP5-857M17 and deleted RP1-310O13 (20q11.21). This supernumerary ring marker had a size of $\sim 4.8 \mathrm{Mb} .{ }^{31}$ Another normal 3-month-old boy was reported to have a $47, \mathrm{XY},+\operatorname{mar}[25 \%] / 46, \mathrm{XY}[75 \%]$ dn karyotype in the amniocytes. The marker was positive for probe RP11-9L7 in 20p12.2. Based on the FISH pattern, the karyotype was interpreted as $\mathrm{r}(20)(: \mathrm{p} 12.2 \sim 12.3 \rightarrow \mathrm{q} 11.1::)$ $[5] / \mathrm{r}(20 ; 20)(:: \mathrm{p} 12.1 \rightarrow \mathrm{q} 11.1:: \mathrm{q} 11.1 \rightarrow \mathrm{p} 12.1::)[2] / \min (20 ; 20)$ $\left(: p 12.1 \rightarrow\right.$ q11.1::q11.1 $\rightarrow$ p12.1:).[1]. ${ }^{37}$ Callier et al. ${ }^{41}$ reported a eutrophic fetus with normal nuchal translucency and no visceral anomaly by ultrasound. The karyotype had a de novo, supernumerary marker, 47,XX,+mar $\operatorname{dn}[12] / 46, \mathrm{XX}[4]$ in $75 \%$ of amniocytes and 54\% of fetal lymphocytes. A 4100 BAC clones (1 Mb) microarray (BlueGnome, Cambridge, UK) showed a duplication of chromosome 20p11.2q11.23. The size of the duplication was $\sim 10.8 \mathrm{Mb}$ from cytoband $20 \mathrm{p} 11.2(25 \mathrm{Mb})$ to 20q11.23 (35.8 Mb). Postmortem examination at 24 weeks of gestation revealed a dysmorphic female fetus. Weight was $670 \mathrm{~g}$ (50th percentile), length was $32 \mathrm{~cm}$ (25th percentile), and head circumference was $22.5 \mathrm{~cm}$ (50th percentile). Dysmorphism included high forehead with frontal bossing, voluminous ocular globes, hypertelorism, low-set ears with hypoplastic helix, wide, flat nasal bridge with bulging alae nasi, long and smooth philtrum, thin upper lip, and microretrognathia. External genitalia examination revealed hypoplastic labia and clitoris. Rockerbottom feet were also noted. There were no visceral malformations, but there were slightly enlarged lateral ventricles. These are the closest matches to our marker, and when all the gains on the short arm and long arm are considered, the phenotype effect may probably be mild and variable based on the level of mosaicism.

In a single-nucleotide polymorphism array mapping of $20 \mathrm{p}$ deletions, 11/21 had smaller deletions ranging between $95 \mathrm{~kb}$ and $4 \mathrm{Mb}$ (chr20:7,383,615-12,746,054) and these individuals had normal development, with no anomalies besides those associated with Alagille syndrome. ${ }^{42}$ The deletion in our patient (chr20:9,493,095-12,089,631) falls within this cohort of deletions with a phenotype corresponding to Alagille syndrome.

Case 10. (Figure 3) A de novo sSMC by aCGH was a gain of $\sim 2.7 \mathrm{Mb}$ from 8p11.21p11.1 (chr8:40,690,198-43,388,233) that was positive for FISH using a RP11-910J4 (8p11.21) probe. The probe gave one signal $(76 \%)$ or two signals (18\%) in cells based on the number of gains on a marker. Gains involving the pericentromeric region of chromosome 8 are reported in individuals typically with developmental delay/mental retardation and nonspecific minor abnormalities. ${ }^{43-45}$

The presence of a large interstitial homozygous region in the long arm of chromosome 8 , not involving the region of copynumber gain, is suggestive of uniparental heterodisomy (UPD8) occurring during meiosis I with two recombination events in the $8 \mathrm{q}$ arm. The coexistence in this patient of a marker chromosome with material from the pericentromeric region of the $8 \mathrm{p}$ arm and the uniparental heterodisomy is a rare occurrence. At least $1.3 \%$ of sSMC cases present with uniparental disomy. ${ }^{21}$ There are previous reported cases of supernumerary chromosomes coincident with mixed uniparental heterodisomy or uniparental isodisomy as well as segmental isodisomy. ${ }^{46}$ There is no known phenotype associated with UPD8. ${ }^{47}$ Therefore, UPD8 most likely does not contribute to the clinical phenotype in this patient, but instead the marker chromosome and potential recessive mutations in genes within the homozygous block can be considered as candidates to explain the phenotype.

Our proband with the marker had mild developmental delay (DQ 76) primarily in the motor areas. The mother underwent amniocentesis for advanced maternal age and positive maternal multiple marker screening for trisomy 21. A small marker chromosome was detected and verified postnatally. Parental karyotypes were normal. The child was delivered by cesarean section at 34 weeks because of gestational hypertension and fetal decelerations. She weighed 1,900 g and was $42 \mathrm{~cm}$ long. Apgar scores were 8 and 8 . She passed newborn screening and had a normal echocardiogram. The mother is G3P2SAB1LC2. The proband's general health has been good. She has normal hearing and vision. No seizures, regression of milestones, hiccups, food intolerance, vomiting, lethargy, rashes, or an unusual odor to the hair, ears, skin, or urine that might suggest an inborn error of metabolism were observed. She attends a special education preschool, where she receives speech therapy. She has made excellent progress in language acquisition. Vital signs were as follows: blood pressure 80/47, pulse 63, height $98.5 \mathrm{~cm}$ (16th centile), weight $15.2 \mathrm{~kg}$ (26th centile), and head circumference $48.5 \mathrm{~cm}$ (23rd centile). She was a verbal child, using only fourand five-word phrases, and had no dysmorphic features.

There has been a suggestion that most prenatally detected SMCs (2/3) are easily identified through conventional cytogenetic and targeted FISH, rendering aCGH unnecessary in these cases. ${ }^{48}$ However, our study demonstrates the usefulness of aCGH on all de novo SMCs. Complex SMCs are recognized more often when aCGH is used. The frequency of complex markers is greater than $0.9 \%,{ }^{10}$ as shown by our small cohort of 10 patients. aCGH drew finer boundaries for the sSMCs to include 5p13 duplication syndrome and 19q11.2q11.3 duplication obesity-related syndrome genes that provided a strong phenotype correlation in the absence of other copy-number changes. sSMCs may sometimes be coincidental, as seen in case 
9 where the patient's Alagille syndrome is related to the JAG1 deletion on the derivative 20. Hence, genome-wide aCGH rules out any compounding factors for the phenotype. Uniparental disomy may be counterintuitive when markers are complex and the centromere and euchromatin are not contiguous.

In addition to markers derived from translocations or from centric fission, de novo markers probably also arise during trisomy rescue by fragmentation and fusion, i.e., intrachromosomal, interchromosomal, interhomolog, and sometimes stabilization with a centromere or duplication. The multiple copy-number changes in case 9 questions if chromothripsis is involved in SSMC formation during trisomy rescue. The simplest marker may have been derived from the trisomy for chromosome 5 being rescued by forming a pericentomeric small ring (case 5). Trisomy 19 is rarely seen in products of conception because it is probably lethal at an early stage, and hence may survive as a marker only following trisomy rescue. The $20 \mathrm{q}$ marker and concomitant derived 20 with a deletion and duplications of $20 \mathrm{p}$ strongly suggest a complex rearrangement between a third chromosome 20 and a normal homolog. A summary of complex markers (Table 2) shows they are most often derived from acrocentric chromosomes. Is this because acrocentric chromosome short arms associate and actively exchange and may shed centromeric fragments that are picked up by fragments formed during trisomy rescue? The chromosome 8 marker was duplicated in a mosaic cell line. This is a common feature observed in neocentromere markers. The duplication probably strengthens the marker's survival by stabilizing the centromere.

Insights on markers. Even a normal aCGH result is useful for classifying the SMCs as benign (case 1) and to rule out other significant copy-number changes in the genome. Detection of low-level mosaics (20-30\%) by aCGH depends on the probe density, distribution, and size of marker. The oligonucleotide and single-nucleotide polymorphism array also detects uniparental disomy in SMC cases (case 10). Because sSMCs are frequently complex, based on our limited sample size, they require rigorous investigation using cytogenetic banding methods ( $G$, $\mathrm{C}$, and AgNOR), followed by whole-genome aCGH analysis and finally FISH for a precise and complete characterization. Chromosome banding (G-, C-, and AgNOR) provides information on morphological features such as acrocentric vs. nonacrocentric, centric vs. acentric and ring vs. marker. The euchromatin DNA content of markers can be determined by aCGH only when copy number changes are localized to the marker by FISH. The identity of the centromere can be assigned only by FISH. Two markers determined to be acrocentric by banding turned out to contain mainly nonacrocentric euchromatin from chromosome 18 and 19 (cases 6 and 7) as determined by aCGH and FISH. However, the chromosome banding guided our focus to copy-number changes in the pericentromeric region of acrocentric chromosomes. Both the acrocentric morphology by banding and gain in copy number in the pericentromeric region of an acrocentric chromosome determined the appropriate FISH probes. Because the majority of the markers are derived from acrocentric chromosomes, some cannot be confirmed by FISH as in case 6, because there are no unique centromeric FISH probes for some acrocentric chromosomes. Also, when the aCGH shows a pericentromere copy-number change as in case 7 , it may be a common copynumber variant, hence, localizing a FISH probe from this region to the marker becomes necessary. In case 9, a very small, highly pyknotic marker determined by banding may have been dismissed as benign. But aCGH revealed multiple chromosome 20 copy-number changes and the corresponding FISH probes localized either to the marker or to a chromosome 20 that appeared apparently normal in G-banding. Only aCGH followed by FISH can determine when sSMC euchromatin is from a different chromosome (cases 6 and 7), noncontiguous chromosome fragments (case 8), or a duplicon in a mosaic cell line (case 10).

\section{SUPPLEMENTARY MATERIAL}

Supplementary material is linked to the online version of the paper at http://www.nature.com/gim

\section{ACKNOWLEDGMENTS}

Kaiser Permanente Southern California institutional review board reviewed the project and approved this work.

The project was conceived and written by K.S.R. using cases reviewed at sign-out; the array work was done by Genedx, and the clinical information was provided by G.T., H.B., and S.A.

\section{DISCLOSURE}

The authors declare no conflict of interest.

\section{REFERENCES}

1. Liehr T, Weise A. Frequency of small supernumerary marker chromosomes in prenatal, newborn, developmentally retarded and infertility diagnostics. Int J Mol Med 2007;19:719-731

2. Graf MD, Christ L, Mascarello JT, et al. Redefining the risks of prenatally ascertained supernumerary marker chromosomes: a collaborative study. J Med Genet 2006:43:660-664.

3. Stankiewicz P, Bocian E, Jakubów-Durska K, et al. Identification of supernumerary marker chromosomes derived from chromosomes 5, 6, 19, and 20 using FISH. J Med Genet 2000;37:114-120.

4. Baldwin EL, May LF, Justice AN, Martin CL, Ledbetter DH. Mechanisms and consequences of small supernumerary marker chromosomes: from Barbara McClintock to modern genetic-counseling issues. Am J Hum Genet 2008;82:398-410.

5. Callen DF, Freemantle CJ, Ringenbergs ML, et al. The isochromosome $18 p$ syndrome: confirmation of cytogenetic diagnosis in nine cases by in situ hybridization. Am J Hum Genet 1990;47:493-498.

6. Crolla JA, Harvey JF, Sitch FL, Dennis NR. Supernumerary marker 15 chromosomes: a clinical, molecular and FISH approach to diagnosis and prognosis. Hum Genet 1995;95:161-170.

7. Huang B, Crolla JA, Christian SL, et al. Refined molecular characterization of the breakpoints in small inv dup(15) chromosomes. Hum Genet 1997;99:11-17.

8. Eggermann K, Mau UA, Bujdosó G, et al. Supernumerary marker chromosomes derived from chromosome 15: analysis of 32 new cases. Clin Genet 2002;62:89-93

9. Mears AJ, el-Shanti H, Murray JC, McDermid HE, Patil SR. Minute supernumerary ring chromosome 22 associated with cat eye syndrome: further delineation of the critical region. Am J Hum Genet 1995;57: 667-673

10. Trifonov $V$, Fluri $S$, Binkert $F$, et al. Complex rearranged small supernumerary marker chromosomes (SSMC), three new cases; evidence for an underestimated entity? Mol Cytogenet 2008;15:1-6. 
11. Vetro A, Manolakos E, Petersen MB, et al. Unexpected results in the constitution of small supernumerary marker chromosomes. Eur J Med Genet 2012;55:185-190.

12. Yu S, Fiedler SD, Brawner SJ, Joyce JM, Zhou XG, Liu HY. Characterizing small supernumerary marker chromosomes with combination of multiple techniques. Cytogenet Genome Res 2012;136:6-14.

13. Liehr T. sSMC homepage. 2005. http://www.med.uni-jena.de/fish/ sSMC/OOSTART.htm.

14. Melo JB, Backx L, Vermeesch JR, et al. Chromosome 5 derived small supernumerary marker: towards a genotype/phenotype correlation of proximal chromosome 5 imbalances. J App/ Genet 2011;52: 193-200.

15. Schinzel A. Catalogue of Unbalanced Chromosome Aberrations in Man, 2nd edn. DeGruyter: New York, 2001.

16. OMIM (Online Mendelian inheritance in man). Johns Hopkins University, Center for Medical Genetics: Baltimore, 1996. http://omim.org/ entry/192500.

17. GeneReviews. http://www.genetests.org.

18. Inherited arrhythimas database. http://www.fsm.it/cardmoc/,Human Genome Mutation Database; https://portal.biobase international.com/ hgmd/pro/search_gene.php?.

19. Stratton RF, DuPont BR, Olsen AS, Fertitta A, Hoyer M, Moore CM. Interstitial duplication 19p. Am J Me.d Genet 1995;57:562-564.

20. Novelli A, Ceccarini C, Bernardini L, et al. Pure trisomy $19 p$ syndrome in an infant with an extra ring chromosome. Cytogenet Genome Res 2005;111:182-185.

21. Liehr T. sSMC homepage. 2006. http://www.med.unijena.de/fish/ ssmc/00start.htm.

22. Vranekovic J, Brajenovic-Milic B, Modrusan-Mozetic Z, Babic I, Kapovic M. Severe psychomotor retardation in a boy with a small supernumerary marker chromosome 19p. Cytogenet Genome Res 2008;121:298-301.

23. Lehman AM, du Souich C, Chai D, et al. 19p13.2 microduplication causes a Sotos syndrome-like phenotype and alters gene expression. Clin Genet 2012;81:56-63.

24. Quack B, Van Roy N, Verschraegen-Spae MR, Klein F. Interstitial deletion and ring chromosome derived from 19q. Proximal 19q trisomy phenotype. Ann Genet 1992;35:241-244.

25. Zung A, Rienstein S, Rosensaft J, Aviram-Goldring A, Zadik Z. Proximal 19q trisomy: a new syndrome of morbid obesity and mental retardation. Horm Res 2007;67:105-110.

26. Davidsson J, Jahnke K, Forsgren M, et al. dup(19)(q12q13.2): array-based genotype-phenotype correlation of a new possibly obesity-related syndrome. Obesity 2010;18:580-587.

27. Hall CE, Cunningham JJ, Hislop RG, Berg JN. A boy with supernumerary mosaic trisomy 19q, involving 19q13.11-19q13.2, with macrocephaly, obesity and mild facial dysmorphism. Clin Dysmorphol 2010;19: 218-221.

28. Oda T, Elkahloun AG, Meltzer PS, et al. Identification of a larger than 3 $\mathrm{Mb}$ deletion including JAG 1 in an Alagille syndrome patient with a translocation t(3;20)(q13.3;p12.2). Hum Mutat 2000;16:92.

29. Database of Genomic Variants. http://projects.tcag.ca/variation.

30. Wanderley HY, Schrander-Stumpel CT, Visser MO, Van Maanen-Op Het Roodt EA, Loneus WH, Engelen JJ. Report of a patient with a trisomy of chromosome region 20q11.2-->20q12 and characterization with FISH. Genet Couns 2005;16:277-282.

31. Guediche N, Brisset S, Benichou JJ, et al. Chromosomal breakpoints characterization of two supernumerary ring chromosomes 20. Am J Med Genet A 2010;152:464-471.
32. Callen DF, Eyre HJ, Ringenbergs $M L$, Freemantle CJ, Woodroffe $P$ Haan EA. Chromosomal origin of small ring marker chromosomes in man: characterization by molecular genetics. Am J Hum Genet 1991;48:769-782.

33. Blennow E, Annerén G, Bui TH, Berggren E, Asadi E, Nordenskjöld M. Characterization of supernumerary ring marker chromosomes by fluorescence in situ hybridization (FISH). Am J Hum Genet 1993;53: 433-442.

34. Batista DA, Escallon C, Blakemore KJ, Stetten G. An accessory marker derived from chromosome 20 and its co-existence with a mosaic trisomy 20 cell line. Prenat Diagn 1995;15:123-127.

35. van Langen IM, Otter MA, Aronson DC, et al. Supernumerary ring chromosome 20 characterized by fluorescence in situ hybridization. Clin Genet 1996:49:49-53.

36. Viersbach R, Engels $H$, Schwanitz G. Identification of supernumerary der(20) chromosomes by FISH in three patients. Am J Med Genet 1997;70:278-283.

37. Crolla JA, Long F, Rivera H, Dennis NR. FISH and molecular study of autosomal supernumerary marker chromosomes excluding those derived from chromosomes 15 and 22: I. Results of 26 new cases. Am J Med Genet 1998;75:355-366.

38. Austin-Ward ED, Castillo S, Dragnic Y, Sanz P, Salazar S, Knoll JH. Clinical findings in a patient mosaic for a supernumerary ring chromosome 20 . Am J Med Genet 2000;91:171-174.

39. Pinto MR, Fonseca e Silva ML, Aguiar J, Quelhas I, Lima MR. Supernumerary ring chromosome 20 in a mother and her child. Am J Med Genet $A$ 2005;133A:193-196.

40. Kitsiou-Tzeli S, Manolakos E, Lagou M, et al. Characterization of a prenatally assessed de novo supernumerary minute ring chromosome 20 in a phenotypically normal male. Mol Cytogenet 2009;2:1.

41. Callier P, Faivre L, Pigeonnat S, et al. Contribution of array CGH in prognosis and genetic counselling of prenatally diagnosed supernumerary ring chromosome 20. Prenat Diagn 2009;29:1002-1005.

42. Kamath BM, Thiel BD, Gai X, et al. SNP array mapping of chromosome 20p deletions: genotypes, phenotypes, and copy number variation. Hum Mutat 2009;30:371-378.

43. Batanian JR, Huang Y, Gottesman GS, Grange DK, Blasingame AV. Preferential involvement of the short arm in chromosome 8-derived supernumerary markers and ring as identified by chromosome arm painting. Am J Med Genet 2000;90:276-282.

44. Anderlid BM, Sahlén S, Schoumans J, et al. Detailed characterization of 12 supernumerary ring chromosomes using micro-FISH and search for uniparental disomy. Am J Med Genet 2001;99:223-233.

45. Brecevic L, Michel S, Starke H, et al. Multicolor FISH used for the characterization of small supernumerary marker chromosomes (sSMC) in commercially available immortalized cell lines. Cytogenet Genome Res 2006;114:319-324.

46. Liehr T, Ewers E, Hamid AB, et al. Small supernumerary marker chromosomes and uniparental disomy have a story to tell. J Histochem Cytochem 2011;59:842-848.

47. Luedi PP, Dietrich FS, Weidman JR, Bosko JM, Jirtle RL, Hartemink AJ. Computational and experimental identification of novel human imprinted genes. Genome Res 2007;17:1723-1730.

48. Gruchy N, Lebrun M, Herlicoviez $M$, et al. Supernumerary marker chromosomes management in prenatal diagnosis. Am J Med Genet A 2008;146A:2770-2776.

49. Tsuchiya KD, Opheim KE, Hannibal MC, et al. Unexpected structural complexity of supernumerary marker chromosomes characterized by microarray comparative genomic hybridization. Mol Cytogenet 2008;1:7.1-11. 